\title{
Digital TRANSFORMATION OF FinANCIAL SERVICES USING ARTIFICIAL INTELLIGENCE, MACHINE LEARNING, AND CLOUd COMPUTING
}

\author{
Prudhvi Parne \\ Information Technology, Bank of Hope, \\ 1655 W Redondo Beach Blvd, Gardena, CA, USA
}

\begin{abstract}
Digital disruption is redefining industries and changing the way business function. Artificial Intelligence is the future of banking as it brings the power of advanced data analytics to combat fraudulent transactions and improve compliance. Financial services are the economical backbone of any nation in the world. There are billions of financial transactions which are taking place and all this data is stored and can be considered as a gold mine of data for many different organizations. No human intelligence can dig in this amount of data to come up with something valuable. This is the reason financial organizations are employing artificial intelligence to come up with new algorithms which can change the way financial transactions are being carried out. Artificial Intelligence can complete the task in a very short period. Artificial intelligence can be used to detect frauds, identify possible attacks, and any other kind of anomalies that may be detrimental for the institution. This paper discusses the role of artificial intelligence and machine learning in the finance sector. Additionally, the paper will provide the necessary strategies that any banking organization can follow when digitizing its operations when implementing Artificial Intelligence, Machine learning and Cloud Computing.
\end{abstract}

\section{KEYWORDS}

Artificial Intelligence, Machine Learning, Finance, Security

\section{INTRODUCTION}

The development of data analysis capabilities has allowed multiple sectors in the industries to identify various beneficial activities to improve efficiency, identify opportunities, develop better capabilities, improve reachability, develop better customer satisfaction, identify the products that can be developed and sustained in a longer run, improve the security measures, and many other benefits accurately implemented to support the business activities.

One of the primary beneficiaries of artificial intelligence and machine learning capabilities can be identified as the financial sectors that are one of the biggest repositories of data that can be explored to identify valuable insights that can be used by the sector to identify opportunities, improve the services, develop better products, provide better customer service capabilities, leverage the artificial intelligence and machine learning capabilities to identify risks, develop automated processes to improve the security measures on the infrastructure and information, and other activities implemented appropriately that can be effectively utilized to improve the business prospects of the financial institute. 


\section{ARTIFICIAL INTELLIGENCE IN FinANCE}

BFSI as it is commonly known stands for Banking, Securities, Finance, and Insurance which forms the core of the financial sector. There are vast amounts of data generated by them because of which systems with high analytical capabilities are required to dig out the crucial information required to grow the business. This knowledge can be also used to make good decisions. Artificial intelligence helps the financial sector by reducing the number of manual errors that were conducted earlier by consistently developing decision-making processes that verify every bit of the information available before the required decisions are established. Machine learning capabilities efficiently utilize artificial intelligence to identify future opportunities by understanding the communication and transactions to develop better strategies to improve the business prospects, identify opportunities, develop autonomous response capabilities to improve the communications with the customers, and many other beneficial activities implemented [1].

\section{IMPORTANCE OF DATA}

The sensitivity of the information available in the financial sector makes it one of the primary targets by attackers to gain access to the infrastructure and the information. Artificial intelligence capabilities established in financial institutes can efficiently identify the possible attacks by developing understanding based on signatures, patterns, anomalies identified, and many other identifiers used to detect abnormal activities in the network to alert the security team on possible intrusion attempts carried out. Mapping various aspects of the information with the historical information to identify the difference in the activities can efficiently identify anomalies in the transactions, analyze the activities of the users in the infrastructure to define activities beyond the roles and responsibilities which may develop into risks to the organizations and identify possible solutions that can be effectively utilized to reduce the security risks [2].

\section{Risk MANAGEMENT}

The financial sector is full of risks daily due to the nature of activities carried out. An organization can efficiently perform by identifying all the risks, identifying the new risks, and define the impact of identified risks in the earliest stage [3]. The business prospects in the Financial Industries are full of risks that need to be identified, protect the organization from higher exposure to risks, identify the risk tolerance and risk appetite of the organization, provide adequate alerts to the risk management team on identified possibilities of risks materializing in the environment, develop all at mechanisms that can appropriately report the risks which can be analyzed by the management to make appropriate decisions based on the data provided by the artificial intelligence and machine learning capabilities that are established to detect activities beyond the acceptable range of the organization to reduce exposure to higher risks which may impact the performance of the organization [5].

Risk management is one of the critical activities in the financial sector and identifying the triggers that may increase the risks for an organization is one of the key activities to be conducted to ensure the exposure in the financial sector by the organization is manageable and under control. The ability of the organization to inspect the live data, learn from the live feed, analyze the available information, identify and detect anomalies that can promote risks, and develop appropriate alert mechanisms and create preventive measures to contain the risk is the key to effectively manage the risks and develop better profitability [3]. 


\section{TOOLS FOR THE FinANCIAL SECTOR}

The financial sectors offer multiple products to the customers with different benefits. The capabilities of the marketing and sales team to promote their products improve the profitability of the organization [4]. Implementation of artificial intelligence and machine learning technologies can enable the organization to improve the business prospects by identifying the prospective customers worldwide, providing proposals for individual tailor-made products that can be provided by the organization, developing conversations and communication with the clients through automated processes, implementation of Chatbots in sales and services can drastically improve the response of the organization consistently to the many queries of the customers with satisfactory responses developed based on the understanding created by the AI/ML process.

An organization can improve the efficiency of responses provided to the customers by implementing artificial intelligence, big data analytical capabilities, and machine learning capabilities to identify the historical information, analyze the information to develop a better understanding, identifying the probable responses that can be provided, implementing appropriate available solutions, identifying the possible combination of solutions that can satisfy the customer needs and also develop profitable business for the organization [2]. The new capabilities established can allow the banks to develop business solutions, standards of implementation, the power of artificial intelligence, the insides of historical information, the ability of the machine working to develop predictability, and other capabilities put together to achieve the best results for the instant services required by the customers to improve their activities.

\section{STRATEgIES TO IMPLEMENT ARTIFICIAL INTELLIGENCE}

To successfully implement artificial intelligence, the banking institution must identify the crosscutting problems that the AI will resolve. This involves breaking down the AI strategy into fragments such as skills and techniques, which will assist in pinpointing challenges, activities, themes, goals and issues that frequently occur across the various AI uses. Identifying the underlying issues is important because the bank is now able to recognize them and administer the most effective and efficient ways to overcome them. The second strategy is aligning the company's data strategy by ensuring that it is up to date with the current technology to minimize data security issues in the future. Therefore, it is essential for banking institutions to prioritize AI that will bring significant changes to the specific areas of the data strategy.

The next strategy to consider is the current legal and ethical issues around AI. Regardless of how the company will decide to use the AI, they must consider consent and data privacy as the main point of contribution. During this strategy, the company will verify to ensure that the AI selected does not leave room for biasness and discrimination and at the same time confirm that the AI remains ethical and practices fairness to the company employees and users. The infrastructure and technology section requires that the bank identify the recurring themes surrounding the company infrastructure and data. In this strategy, the company will be able to identify the four essential layers of data, thus recognizing the technology that will be appropriate for each layer. These layers include communicating insights from data, data processing, data storage and gathering of data.

The next step is determining the capacity and skills required during AI implementation. This helps identify the existing skill gaps that apply to various business cases, such as providing the training requirements or even hiring new staff or collaborating with an AI provider. Additionally, during the implementation of the strategy, the bank institution will change the current management if the need arises to ensure that the incoming leader will provide customers and 
employees will be able to be impacted positively by being availed with the proper engagement and communication technique.

\section{STRATEgIES TO IMPLEMENT MAChINE LEARNING}

Planning and collaboration are key factors to establishing machine learning in any institution. Using any technology insertion, vision starts and moves on to execution followed by incessant monitoring and development. Building an ML implementation plan can be described in few steps. The first step is establishing a vision. This is a very important step in implementing new technology. When business and Information come together intending to establish a vision and define clear objectives for an ML implementation. All the objectives should be simple as improving the correctness of the fraud detection mechanism to ensuring that overall operations efficiency, but also it requires business and Information Technology alignment and agreement to work towards one target. To measure success, it is very important to understand what you want to achieve. The most common use cases by searching for places that are repetitive and laborintensive such as configuration management, systems updates, image classification, and optimizing the data center operations. The value of the business is also measured in this category to establish key performing indicators.

The second step is defining data requirements since it's the single most important element needed for machine learning and implantation to be successful. Feeding, collecting, and storing the systems huge amounts of data that is reliable to ensure the accuracy of the machine learning algorithms. The process required by the data management includes providing an original set of historic data to teach the ML processing strategies, continuous data insertion to improve and also train the accuracy of the models. Beyond the initial training phase, better infrastructure will be needed to gather new data from which to learn over some time.

Another strategy is setting up the management process to prevent technology insertions that frequently fail following enough change management procedures. Change administration and training are two chief aspects of delivering acceptance on a significant scale effort, and machine learning, on the other hand, is no different. In Machine learning, change management comprises making reflections on the current business procedures and reengineering them based on the updated business models. Similarly, the training programs cover product features, the company objectives, and the freshly innovated procedures that are imperative to establish collective awareness and support for the company and its objectives and increase the efficiency of its operations. The last strategy is developing revalidation and monitoring, which is executed to enhance the success of a particular application and assess whether it needs changes established by measuring its corresponding value. To ensure that Machine Learning models remain relevant after implementation, they must be consistently validated and updated. To attain this, the organization needs to guarantee that the implementation plan comprises an updated version based on providing outcomes and integrating into the existing systems by intensifying the quantities of data. Similarly, the organization should also measure how the ML algorithm will affect the company goals on a broader basis.

\section{Strategy to ImPlement Cloud COMPUTING}

Evaluating the current information technology state is the first thing to do to start building a cloud strategy. All the ins and outs of information technology should be understood. For instance, the latest information technology state should consist of non-value-add capabilities, a long-time value, expensive to have and keep safe, and not always firms aligned. Also, it is essential to consider the institution's capabilities regarding information technology. There are some factors to 
consider determining whether it's the correct position to invest in the cloud, such as considering the organizations size, the regional and world footprint, and if the business is established or a startup. The following strategy is to implement cloud computing in the desired state. After evaluating the current information technology state, consider what an ideal information technology condition looks like. The desired state might focus on core competencies, low upfront cost, and very risky security certificates build in and improve time to value. Also, understanding gaps is among the strategies to implement cloud computing. Identifying gaps that are between the current and desired states is easy. Some examples of gaps that are used include security concerns, cost discrepancy, and firms being focused.

Nevertheless, developing initiatives. Both technical and business initiatives can be developed to allows to bridge the gap. Examples of such initiatives include reducing the cost of information technology infrastructure, adapting quickly to the changes in the market, and acquiring a competitive advantage in the marketplace. Finally, the bottom line is that before jumping into cloud adoption, it is advised to know the drivers for the firm to move to the cloud and the best solutions required by the drivers. A solid and sound cloud strategy will be based on outlining the solutions that are already existing, defining the expected outcome and building initiatives that are based on the gap.

\section{BENEFACTORS OF ARTIFICIAL INTELLIGENCE AND MACHINE LEARNING}

The aggregate potential cost savings for banks from AI applications is estimated at $\$ 447$ billion by 2023 , with the front and middle office accounting for $\$ 416$ billion of that total [7].

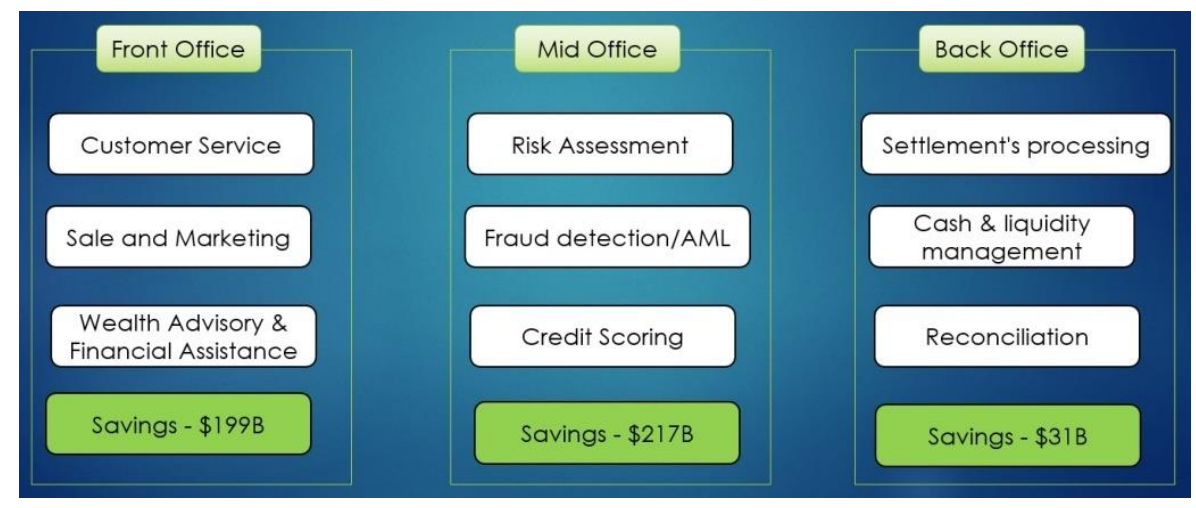

One of the primary beneficiaries of the advanced technologies used in the security measures on the information by implementing blockchain technologies can also use analytical capabilities on the secured information to efficiently conduct analytical capabilities with an accuracy of information, authenticity, and accuracy of the information without being adulterated. Blockchain has allowed higher security measures to be implemented on the information in transit and storage, data analytics capabilities have improved the decision-making capabilities, artificial intelligence improves the decision-making process by identifying media's insights from the historical information that can be applied, and the machine-learning capabilities evolve their understanding on the live information feed to develop the understanding instantly and identify the patents in the information that can be effectively utilized to develop accurate responses which can be accomplished automatically and with high accuracy [4].

On other important aspects in the financial industry is the regulator the aspect due to the sensitivity of the information and the high risks which requires the regulatory authorities to implement stringent rules and regulations to secure the interest of the investor, protect the information privacy, develop ethical practices by the organizations, and provide adequate 
suggestions on security implementation to the organizations implementing infrastructure and business activities. With the increased number of cyber incidents daily, the natural response from the regulatory authority to implement stricter measures to protect the interest of the citizens, to develop accountability and responsibilities assigned to promote ethical behavior, and provide judicial capabilities to resolve disputes, information protection, information privacy, and accountability of activity are mandated by regulatory authorities worldwide. An organization must abide by the rules and regulations and identify various breach incidents in the initial stages to improve the implementation of rules and regulations to protect the interest of the investor and protect the infrastructure from unauthorized activities [5].

\section{EFFICIENT AND EFFECTIVE UTILIZATION OF TECHNOLOGY}

Identifying the consistent utilization of artificial intelligence and machine-learning capabilities which may reduce the number of Manual jobs but provide consistency in the information and its authenticity, developing appropriate unbiased and decision-making capabilities, identifying the opportunities to diversify and improve business prospects, develop identification of future requirements of the customers in the financial sectors, understanding the needs of the customers, developing autonomous conversation capabilities by the organization with its existing and prospective customers can greatly improve the opportunities for the organization to improve the business prospects and develop better profitability [2].

Identifying the historical value of the information, developing analytical capabilities on the existing information, understanding the hidden value of the information available, using the value to develop better capabilities, improving the processes, discovering new processes, implementing improvements in customer services, improving the cybersecurity measures, enhancing the risk management process to detect risks at the earliest age, interacting with the customers to provide solutions available in the organization, and many other beneficial activities can be effectively implemented with the use of the data analytical capabilities, artificial intelligence implementation, and machine-learning processes established that can effectively identify, understand, develop responses accurately, improves the responses based on the feedback received to provide best solutions to the customers and improve the profitability of the organization consistently [3].

Firms can utilize AI to obtain more data from meager verifiable models or recognize non-direct connections in the request stream. Machine learning can be used to make 'trading robots' that then, at that point, show themselves how to respond to market changes. Market sway analysis includes assessing the impact of an association's trading on market costs. Since firms are worried about the effect of exchanges, particularly massive sales, on market costs, a more precise assessment of this effect is vital to timing exchanges and limiting trading execution costs. Firms are exploring utilizing AI tools to evaluate the market effect of a given business. The impact of an association's trading on market costs is famously tricky to show, particularly for less fluid protections, where information on similar past exchanges is scant. AI and machine learning can supplement traditional market sway models[2].

AI tools may help by enlarging models effectively or acquiring a machine learning approach to limit the trading sway on costs and liquidity. For the most dynamic efficient assets, as much as $66 \%$ of the gain on exchanges is assessed to be lost to market sway costs [6]. AI tools may help by enlarging models effectively being used, or by acquainting a machine learning approach with limit the trading sway on prices and liquidity for trading both into and out of enormous market positions, or as a piece of consistently trading strategies. Machine learning is frequently used to distinguish gatherings of bonds that act comparatively to one another. Like this, they can depend on more information focuses, giving better gauges of value developments when the market is 
dainty. The subsequent apparatus bunches bonds into wide, naturally comparative containers. Afterward, utilizing group analysis, gather the most tantamount items together in each pail to score the liquidity of individual bonds [5].

Additionally, AI can assist with distinguishing how the circumstance of exchanges can limit market sway. Market sway models can be fostered that depict how the impact of business relies upon past interactions as a beginning stage. The models endeavor not to plan trades too intently together to try not to have a market sway more prominent than the number of its parts [6]. These models can set out the ideal trading plans for a scope of situations and afterward change the timetable as the genuine exchange advances, using managed learning strategies to make the transient predictions deciding those changes.

\section{Case Study: AI ANd ML BaSed Applications}

In Insurance domain, AI and machine learning applications can significantly increase some protection area capacities, such as endorsing and preparing claims. In supporting, AI frameworks dependent on NLP can grow enormous business guaranteeing and life or inability endorsing. These applications can gain from training sets of past claims to feature critical contemplations for human decision-creators [4]. Machine learning procedures can decide repair costs and naturally sort the seriousness of vehicle mishap harm. Moreover, AI might help diminish claims preparing times and functional expenses. Insurance agencies are additionally investigating how AI and machine learning and remote sensors (associated through the 'web of things) can distinguish, and sometimes forestall, insurable episodes before they happen, for example, compound spills or auto crashes [2].

Credit scoring tools/applications that utilize machine learning are intended to accelerate loaning decisions while possibly restricting gradual danger. Since a long time ago, Loan specialists have depended on credit scores to settle on loaning decisions for firms and retail customers. Information on exchange and installment history from monetary organizations generally became the establishment of most credit scoring models. These models use tools like relapse, decision trees, and measurable analysis to produce a credit score utilizing restricted measures of organized information. In any case, banks and different moneylenders are progressively going to extra, unstructured, and semi-organized information sources, including online media movement, cell phone use, and instant message action, to catch a more nuanced perspective on creditworthiness and further develop the rating exactness of advances. Applying machine learning algorithms to this star grouping of new information has empowered the evaluation of subjective factors like customer conduct and pay [1].

The capacity to use extra information on such measures considers a more prominent, quicker, and less expensive division of borrower quality and eventually prompts a fast credit decision. Be that as it may, the utilization of individual information raises other policy issues, including those identified with information security and information insurance [5]. As well as working with a conceivably more exact, divided evaluation of credit worthiness, the utilization of machine learning algorithms in credit scoring might empower more noteworthy admittance to credit [4]. In conventional credit scoring models utilized in specific markets, a potential borrower should have an adequate measure of recorded credit data available to be considered 'scorable.' without this data, a credit score can't be created, and a conceivably creditworthy borrower is frequently unfit to obtain credit and assemble a credit history. With the utilization of elective information sources and the use of machine learning algorithms to assist with fostering an evaluation of capacity and ability to reimburse, moneylenders might have the option to show up at credit decisions that beforehand would have been outlandish. While this pattern might profit economies with shallow credit markets, it could prompt non-sustainable expansions in credit exceptional in nations with 
profound credit markets. For the most part, it has not yet been demonstrated that machine learning-based credit scoring models beat customary ones for evaluating creditworthiness [5].

There are a few benefits and disservices to utilizing AI in credit scoring models. AI permits enormous measures of information to be dissected rapidly. Therefore, it could yield credit scoring arrangements that can deal with a more extensive scope of credit inputs, bringing down the expense of surveying credit hazards for specific people and expanding the number of people for whom firms can gauge credit hazards [5]. An illustration of the use of enormous information to credit scoring could incorporate the evaluation of non-credit charge installments, for example, the convenient installment of wireless and other service bills, in the mix with different information. Also, individuals without credit history or credit score might have the option to get an advance or a credit card because of AI, where an absence of credit history has customarily been a constraining element as option pointers of the probability to reimburse have been inadequate in ordinary credit scoring models [1].

Notwithstanding, the utilization of complex algorithms could bring about an absence of straightforwardness to shoppers. This 'discovery' part of machine learning algorithms may thus raise concerns. When utilizing machine learning to allot credit scores to settle on credit decisions, it is, for the most part, harder to give buyers, inspectors, and directors a clarification of a credit score and coming about credit decisions whenever tested [6]. Furthermore, some contend that using new elective information sources, like online conduct or non-conventional monetary data, could bring predisposition into the credit decision. In particular, purchaser support bunches call attention to that machine learning tools can yield blends of borrower qualities that foresee race or sexual orientation, factors that fair loaning laws disallow considering in numerous locales. These algorithms may rate borrowers from an ethnic minority at a greater danger of default because comparable borrowers have customarily been given less ideal credit conditions. The availability of chronicled information across a scope of borrowers and credit items is critical to an exhibition of these tools. Moreover, the availability, quality, and dependability of information on borroweritem execution across a broad scope of monetary conditions are likewise crucial to display these danger models. Again, the absence of information on new AI and machine learning models, and the absence of data about the collection of these models in an assortment of monetary cycles, has been noted by certain specialists [3].

\section{Conclusion}

Artificial Intelligence and Machine Learning are groundbreaking technologies that are still in their primary stages of development and adoption. They have very high capabilities and if they are implemented correctly can change the very way the finance sector is currently operating. This is the reason, almost all the big financial organizations are investing heavily in these technologies because the return on investments is very high. There is a lot of hope riding on these technologies and the IT services companies are trying to deliver them with utmost accuracy. There is still some time required for these technologies to mature and recognize their utmost potential. Companies need to understand that these technologies must be used to make the lives of employees easy. They should not be a reason to replace the human workforce because nothing can beat the human instincts which are required in the financial sector. The management of these financial organizations needs to come up with the best combination of human and artificial intelligence for the development of the industry, mankind, and the world. 
International Journal of Artificial Intelligence \& Applications (IJAIA), Vol.12, No.6, November 2021

\section{APPENDIX}

\section{REFERENCES}

[1] Lee, J. (2020). Access to Finance for Artificial Intelligence Regulation in the Financial Services Industry. Springer Link Articles 731-740.

[2] Joseph, B. \& Collins, P. D. (2021). Adversary-Aware Learning Techniques and Trends in Cybersecurity. Springer Publishing 17-36.

[3] John, G., \& Mooney, M. C. (2018). Disrupting Finance - FinTech, and Strategy in the 21st Century. Springer Publishing, 33-50.

[4] Itay, G. \& Jagtiani, J. \& Klein, A, (2018). Philadelphiafed 2018 fintech conference, Fintech and the new financial landscape , 4.

[5] Raghad, G. L. (2019). The Application of Artificial Intelligence in Financial Compliance Management. ACM Article, 1-6.

[6] FSB (2017), https://www.fsb.org/2017/11/artificial-intelligence-and-machine-learning-in-financialservice/, 5-20.

[7] BusinessInsider (2021)https://www.businessinsider.com/ai-in-banking-report

\section{AUTHOR}

Prudhvi Parne received the Master's (MS) degree in Computer Science from University of Louisiana, Lafayette, LA, USA. His expertise spans in the areas of Cloud Architecture, Software Development, Finance, Banking, Hybrid clouds, Product Management, and Product leadership.

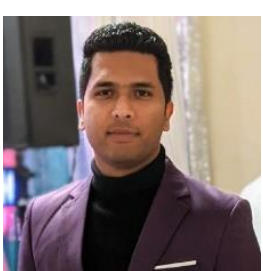

